\title{
Erratum to: Symplectic embeddings of polydisks
}

\author{
R. Hind ${ }^{1}$ - S. Lisi ${ }^{2}$
}

Published online: 2 December 2016

(C) Springer International Publishing 2016

\section{Erratum to: Sel. Math. New Ser. (2015) 21:1099-1120 DOI 10.1007/s00029-013-0146-2}

The original proof of Lemma 6.1 implicitly assumed that all upper level curves in the limiting building $F$ had punctures asymptotic to geodesics in the classes $(a, 0)$. (Throughout we are adopting the same notation as in the main paper.) The purpose of this erratum is to justify this assumption; the justification will rely on our quantitative hypotheses. The statement of the lemma remains unchanged.

Suppose that $F_{0}$, the top level of our building, has a single curve $f$ which is not a cover of curves in the foliation $\mathcal{F}$. Then since $F$ has genus 0 , each puncture of $f$ must be matched to unions of limits of the foliation curves. Hence the limiting geodesics must indeed lie in the classes $(a, 0)$. It therefore suffices for us to establish the following.

Lemma 0.1 $F_{0}$ contains a single curve that is not a cover of a curve in the foliation $\mathcal{F}$.

Proof Observe first that since the space of closed foliation curves in $\mathcal{F}$ has two connected components, and the building $F$ is a limit of curves having intersection number

The online version of the original article can be found under doi:10.1007/s00029-013-0146-2.

$凶$ R. Hind

Richard.K.Hind.1@nd.edu

S. Lisi

samuel.lisi@univ-nantes.fr

1 Department of Mathematics, University of Notre Dame, Notre Dame, IN, USA

2 Department of Mathematics, Université de Nantes, Nantes, France

Birkhäuser 
1 with the fiber (foliation) class, by positivity of intersection, there can be at most two curves in $X \backslash L$ that are not in our original foliation. Arguing by contradiction we assume there are two curves and denote them by $f_{1}$ and $f_{2}$. Note that $f_{1}$ and $f_{2}$ must be somewhere injective since they have intersection number 1 with certain closed foliation curves. Let $d_{i}=f_{i} \bullet \mathbb{C} P^{1}(\infty)$ and $e_{i}=f_{i} \bullet E$.

Let $C_{0} \cup C_{1}$ be a broken configuration in the foliation $\mathcal{F}$, as in Proposition 5.1 and Lemma 5.2. If either curve $f_{i}$ has a puncture asymptotic to a Reeb orbit covering a geodesic in class $(a, 0)$ for $a>0[a<0]$, then $f_{i} \star C_{0} \geq 1\left[f_{i} \star C_{1} \geq 1\right]$ and thus $f_{i} \bullet C \geq 1$ for every closed curve $C$ in our foliation. This is a contradiction since the total intersection number of $F$ with each $C$ is 1 .

Thus, $f_{1}$ and $f_{2}$ must converge at each of their punctures to an orbit representing a class $(k, l)$ with $l \neq 0$. As $F$ has genus 0 and these punctures cannot be matched with curves in the foliation, it follows then that $f_{1}$ and $f_{2}$ must both be planes. Denote the homology classes of their asymptotic limits by $\left(k_{1}, l\right)$ and $\left(k_{2},-l\right)$. Without loss of generality, we assume $l \geq 1$.

Now we compare the Fredholm indices of the various components.

The top level consists of $f_{1}$ and $f_{2}$, together with some number of planes that cover curves of type $C_{0}$ and $C_{1}$ (defined as in Proposition 5.1). Let $K_{0}$ and $K_{1}$ be the numbers of planes that cover curves of type $C_{0}$ and $C_{1}$ respectively and let $a_{i}, i=1, \ldots, K_{0}$ and $b_{i}, i=1, \ldots, K_{1}$ be their covering multiplicities, and $r_{i}, i=1, \ldots, K_{0}$ and $s_{i}$, $i=1, \ldots, K_{1}$ be the respective numbers of ends. By Proposition 3.1 such covers have Fredholm indices

$$
\text { index }=r_{i}-2+2 a_{i} ; \quad \text { index }=s_{i}-2+2 b_{i}
$$

respectively.

The bottom levels consist of a sub-building in $T^{*} L$, passing through the $2 d$ constraint points. The total Fredholm index is $2 S-2 K$ where $S$ is the number of positive ends and $K$ is the number of components. Since the sub-building must satisfy $2 d$ point constraints, we may assume $2 S-2 K \geq 4 d$. (This holds even if some components are multiply covered.)

Finally, the total Fredholm index of the whole building (omitting point constraints) is $4 d$. We compute this as the sum of the indices of the components of the building, subtracting constraints from matching asymptotics:

$$
\begin{aligned}
4 d= & (2 S-2 K)+\operatorname{index}\left(f_{1}\right)-1+\operatorname{index}\left(f_{2}\right)-1 \\
& +\sum_{i=1}^{K_{0}}\left(\left(r_{i}-2+2 a_{i}\right)-r_{i}\right)+\sum_{i=1}^{K_{1}}\left(\left(s_{i}-2+2 b_{i}\right)-s_{i}\right) .
\end{aligned}
$$

Then since $2 S-2 K \geq 4 d$ and the sums are clearly nonnegative, we see that the equality is possible only if (index $\left.\left(f_{1}\right)-1\right)+\left(\operatorname{index}\left(f_{2}\right)-1\right) \leq 0$. However, the $f_{i}$ are somewhere injective planes, and by Proposition 3.1 they have nonnegative odd index, so index $\left(f_{1}\right)=\operatorname{index}\left(f_{2}\right)=1$. 
Now using Proposition 3.1 again for the index formula we observe

$$
\begin{aligned}
0<\operatorname{area}\left(f_{2}\right) & =R d_{2}-e_{2}+k_{2}-2 l \\
& =(R-3) d_{2}-l+3 d_{2}-e_{2}+k_{2}-l \\
& =(R-3) d_{2}-l+\frac{1}{2}\left(\operatorname{index}\left(f_{2}\right)+1\right) \\
& =(R-3) d_{2}-l+1 .
\end{aligned}
$$

Note that $d_{2} \geq 0$ and $R-3<0$, so $0<\operatorname{area}\left(f_{2}\right) \leq 1-l$. As we assume $l \geq 1$ this gives our contradiction. 